\title{
UNIVERSITYOF
}

FORWARD

THINKING

WESTMINSTER用

WestminsterResearch

http://www.westminster.ac.uk/westminsterresearch

Validation of a fast method for quantification of intra-abdominal and subcutaneous adipose tissue for large-scale human studies

Borga M, Thomas EL, Romu T, Rosander J, Fitzpatrick J, Leinhard OD, Bell JD

This is the accepted version of the following article: Borga M, Thomas EL, Romu $T$, Rosander J, Fitzpatrick J, Leinhard OD, Bell JD (2015) Validation of a fast method for quantification of intra-abdominal and subcutaneous adipose tissue for large-scale human studies NMR in Biomedicine 28 (12) 1747-1753 1099-1492

Which has been published in final form at https://dx.doi.org/10.1002/nbm.3432

The WestminsterResearch online digital archive at the University of Westminster aims to make the research output of the University available to a wider audience. Copyright and Moral Rights remain with the authors and/or copyright owners.

Whilst further distribution of specific materials from within this archive is forbidden, you may freely distribute the URL of WestminsterResearch: ((http://westminsterresearch.wmin.ac.uk/).

In case of abuse or copyright appearing without permission e-mail repository@westminster.ac.uk 
Validation of a Fast Method for Quantification of Intra-abdominal and

Subcutaneous Adipose Tissue for Large Scale Human Studies

Running Title: Quantification of IAAT and ASAT

Magnus Borga ${ }^{1,2,5}$, E Louise Thomas ${ }^{3}$, Thobias Romu ${ }^{1,2}$, Johannes Rosander ${ }^{5}$, Julie Fitzpatrick $^{3}$, Olof Dahlqvist Leinhard ${ }^{3,4,5}$, Jimmy D Bell ${ }^{3}$

1. Department of Biomedical Engineering, Linköping University, Sweden

2. Centre for Medical Image Science and Visualization (CMIV), Linköping University, Sweden

3. Department of Life Sciences Faculty of Science and Technology University of Westminster, London, United Kingdom

4. Department of Medical and Health Sciences, Linköping University, Sweden

5. Advanced MR Analytics AB, Badhusgatan 5, SE-582 22 Linköping, Sweden

\section{Author of Correspondence}

Professor Jimmy D Bell, Department of Life Sciences, Faculty of Science and Technology, University of Westminster, 115 New Cavendish Street, London W1W 6UW

Tel: 02035064608

Email: J.Bell@westminster.ac.uk

Word Count $-3,543$

Keywords: adipose tissue, fat quantitation, obesity, MRI, Dixon, abdominal fat

Abbreviations: IAAT: intra-abdominal-adipose tissue; ASAT: abdominal subcutaneous adipose; TF: trunk-fat; AMRA: Advance MR Analytics; CoV: coefficient of variation 



\section{Graphical abstract}

In this study we tested the value of using the semi-automated fat-muscle-quantitation system (AMRA ${ }^{T M}$ Profiler) in the analysis of MR images for population studies. The results show extremely high agreement with the current "gold-standard" method across a range of BMI, with the $A M R A^{T M}$ Profiler technique taking up to 10-fold less time compared to its counterpart. The speed and robustness of this method makes it an ideal tool for small and large-scale human phenotypic studies

Whole Body T1-Weighted Images

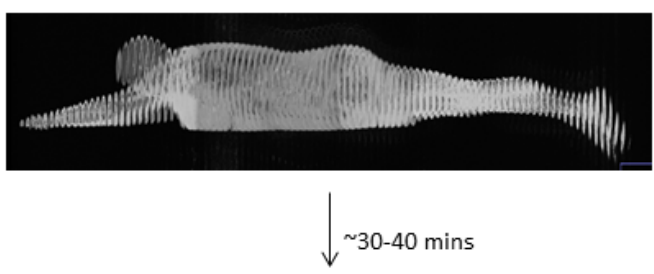

SliceOmatic Analysis of Abdomen
3D Dixon acquisition for AMRATM Profiler

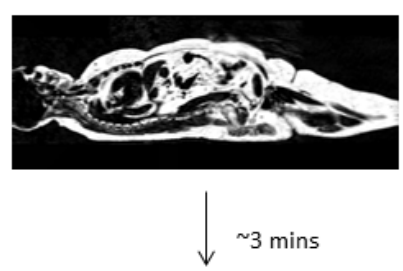

AMRA $^{\text {TM }}$ Profiler analysis
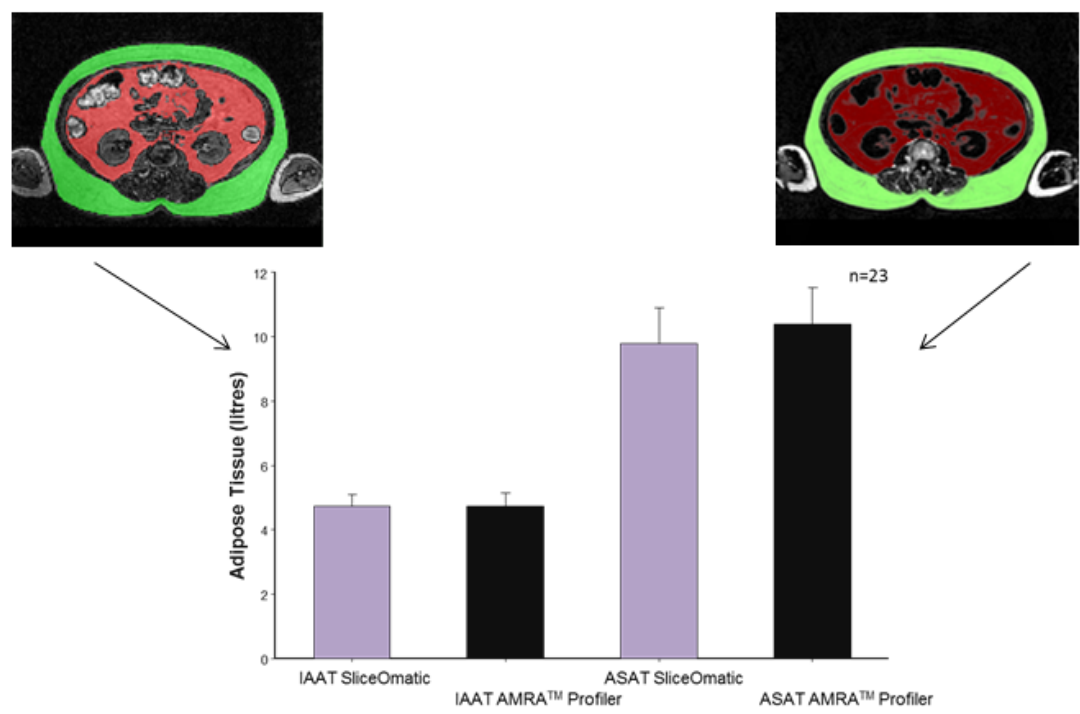


\section{Abstract summary}

Central obesity is the hallmark of a number of non-inheritable disorders. The advent of imaging techniques such as magnetic resonance imaging (MRI) has allowed for a fast and accurate assessment of body fat content and distribution. However, image analysis continues to be one of the major obstacles for the use of MRI in large scale studies. In this study we assess the validity of the recently proposed fat-musclequantitation-system (AMRA ${ }^{\mathrm{TM}}$ Profiler) for the quantification of intra-abdominal adipose tissue (IAAT) and abdominal subcutaneous adipose tissue (ASAT) from abdominal MR images. Abdominal MR images were acquired from 23 volunteers with a broad range of BMIs and analysed using SliceOmatic, the current goldstandard, and the $\mathrm{AMRA}^{\mathrm{TM}}$ Profiler based on a non-rigid image registration of a library of segmented atlases. The results show that there was a highly significant correlation between the fat volumes generated by both analysis methods, (Pearson correlation $r=0.97 p<0.001$ ), with the $\mathrm{AMRA}^{\mathrm{TM}}$ Profiler analysis being significantly faster ( $\sim 3$ mins) than the conventional SliceOmatic approach ( $\sim 40$ mins). There was also excellent agreement between the methods for the quantification of IAAT (AMRA $4.73 \pm 1.99$ vs SliceOmatic $4.73 \pm 1.75$ litres, $p=0.97)$. For the AMRATM Profiler analysis, the intra-observer coefficient of variation was $1.6 \%$ for IAAT and $1.1 \%$ for ASAT, the inter-observer coefficient of variation was $1.4 \%$ for IAAT and $1.2 \%$ for ASAT, the intra-observer correlation was 0.998 for IAAT and 0.999 for ASAT, and the inter-observer correlation was 0.999 for both IAAT and ASAT. These results indicate that precise and accurate measures of body fat content and distribution can be obtained in a fast and reliable form by the AMRA ${ }^{T M}$ Profiler, opening up the possibility of large-scale human phenotypic studies. 


\section{Introduction}

Today, it is generally recognized that central obesity is a key risk factor for the development of a number of metabolic disorders $(1,2)$. It has also been shown that indirect measures such as body-mass index (BMI), waist circumference, and bioimpedance are poor predictors of regional body fat distribution on an individual level (3-5). The advent of tomographic imaging modalities such as computertomography $(\mathrm{CT})$ and magnetic resonance imaging (MRI) has greatly facilitated our ability to directly measure body fat content and distribution in an accurate and reproducible manner $(6,7)$. Currently, it is possible to obtain a whole body MRI scan of an individual, in less than 5 minutes, allowing total and regional fat depots to be measured in detail. However, scan costs and image analysis are still substantial obstacles for large population studies. Indeed, MRI measures of whole-body regional body fat distribution have up to now been limited to research studies in relatively small cohorts of subjects, for example 80 subjects in (7) and less than 500 in (5). In studies with larger cohorts such as the Dallas Heart Study (8), the Framingham Heart Study (9) and the Multi-Ethnic Study of Atherosclerosis (MESA) (10), very limited abdominal MR/CT imaging was carried out, with some studies using single-slice to define abdominal adiposity. This clearly puts considerable limitations on the total information available to researchers (11). Furthermore, although cost per scan has reduced considerably in recent years, the time required to analyse these datasets continues to be a major limiting factor, mainly due to the lack of fast, reliable and reproducible methodologies (12). 
In the last few years, an increasing number of genome-wide association studies (GWAS), using ever-larger cohorts, have been carried out in order to identify common genetic variants associated with complex diseases including obesity, diabetes, heart disease and cancer (13). In many of these studies, phenotyping of subjects was limited to either weight or BMI, with some using also waistcircumference. More recently a number of large population studies have been initiated where more in-depth phenotyping is sought, including the use of MRI and Dual-energy X-ray absorptiometry (DXA) methodologies, for the purpose of measuring body-fat distribution. One example is the UK Biobank, where up to 100,000 individuals will be scanned using MRI, while in the German Cohort Biobank it is envisaged that 30,000 volunteers will be scanned, many of whom will be followed longitudinally. Besides the obvious demand for efficient scanning protocols, the resulting millions of images need to be analysed in a fast and reproducible manner and at a minimum cost. Although existing manual and semi-automated systems can be used to analyse images from small cohorts ( $<100$ subjects), they are not feasible for use in large population studies.

The aim of this study is to evaluate the performance of a rapid semi-automated tool for quantification of body fat, including intra-abdominal-adipose tissue (IAAT) and abdominal subcutaneous adipose tissue (ASAT) volumes from MR images. Recently, a number of such methods have been proposed (14-20). Most of these methods (1419) rely on binary classification of adipose tissue, making them sensitive to partial volume effects (21), a problem that increases with lower spatial resolution. In contrast to these methods, a new method has been proposed, AMRATM Profiler, based on quantitative fat imaging where the entire fat signal within a certain compartment is taken into account $(22,23)$. In addition to reducing the sensitivity to partial volume 
effects, this makes the method less sensitive to segmentation errors. Also in (19), a similar approach was used where fat fraction was integrated within the segmented regions. In Würslin et al. (20) a fuzzy segmentation of T1-weighted spin-echo images was used to alleviate the problem of partial volume effects. In both these approaches, however, the segmentation was based on a 2-dimensional slice-by-slice analysis. The method used in this study is based on a true 3-dimensional analysis using atlas-based segmentation.

The qualities of the proposed method make it an ideal tool for potential large-scale human studies. However, this tool has not been fully validated against the current gold-standard technique for fat quantitation of MR images. Here, the AMRA ${ }^{T M}$ Profiler is assessed against the tool of choice for fat quantitation, the commercially available SliceOmatic. The results show excellent agreement between the methods across a range of $\mathrm{BMI}$, with analysis using the $\mathrm{AMRA}^{\mathrm{TM}}$ Profiler taking up to 10 times less compared with SliceOmatic. The speed and robustness of the AMRA ${ }^{\mathrm{TM}}$ Profiler makes it the ideal tool for small and large-scale human phenotypic studies. 


\section{Materials and Methods}

\section{Image Acquisition}

Written, informed consent was obtained from all volunteers. Ethical permission for this study was obtained from the research ethics committee of Hammersmith and Queen Charlotte's and Chelsea Research Ethics Committee Hospital, London (Rec: 07Q04011/19). In total, 23 volunteers (12 male, 11 female) were recruited via advertisements in newspapers, websites, and academic newsletters, inviting male and female volunteers from the general public. No age constraints were placed on recruitment in order to generate cross-sectional data. Average BMI $31.7 \pm 5.1 \mathrm{~kg} / \mathrm{m}^{2}$ (range 22-46 kg/m²); age 36-66 years. Each subject was scanned with two different protocols on a 1.5T multinuclear scanner (Achieva, Philips Medical Systems, Best, The Netherlands).

\section{Scanning Protocol}

Two different MRI acquisition protocol were used in this study in order to maximise the capabilities of each analysis tool.

Established T1-weighted Acquisition Protocol for SliceOmatic: The first scan was obtained using a rapid T1-weighted protocol as previously described (6). Briefly, a whole-body axial T1-weighted spin echo sequence was acquired using a body coil and no respiratory gating (typical parameters: repetition time $560 \mathrm{~ms}$, echo time 18 ms, slice thickness $10 \mathrm{~mm}$, inter-slice gap $10 \mathrm{~mm}$, flip angle $90^{\circ}$, number of excitations 1). Images were acquired as nine equal stacks of twelve slices at the isocentre of the magnet with the subjects in prone position. 
3D Dixon acquisition for AMRA ${ }^{\mathrm{TM}}$ Profiler: The second scan was carried out using a phase-sensitive multi-point 3D Dixon acquisition (24) with coverage from the neck down to the knees using the integrated quadrature body coil. In this scan, the subjects were in a supine position. 7 image stacks were acquired, of which stacks number 2-5, covering the abdomen, were acquired during breath hold (17 s). Parameters for the image acquisition were as follows: repetition time $5.86 \mathrm{~ms}$ (8.16 ms for stacks 3-4 covering the liver), echo time $n \times 1,15 \mathrm{~ms}, n=1-4$ ( $n=1-6$ for stacks 3-4), matrix size, $172 \times 158$, slice thickness $4.2 \mathrm{~mm}$ (5 mm for stack 7 covering lower part of thigh) and flip angle $13^{\circ}$.

Given that each acquisition protocol resulted in slightly different anatomical coverage, for a robust comparison therefore it was decided to extract the same area from both datasets, using the previously published definition of the abdominal area: 'from the image containing the femoral heads, to the slice containing the top of the liver/bottom of the lungs' (6).

\section{Image Analysis}

\section{Reference Method (SliceOmatic)}

The T1-weighted images were analysed as previously described using the semiautomated software SliceOmatic (Tomovision, Montreal, Quebec, Canada) which has become the leading tool for analysis of body-fat in clinical and pre-clinical research (8). Briefly, total and regional volumes were recorded in litres (I); comprising; abdominal-subcutaneous adipose tissue (ASAT) and intra-abdominal adipose tissue (IAAT) (25). As previously stated the abdominal region was defined as the image slices from the slice containing the femoral heads, to the slice containing the top of the liver/bottom of the lungs (6); therefore the measurement of IAAT contains a 
mixture of visceral, perirenal, and retroperitoneal adipose tissue. In order to gauge abdominal adiposity as a whole, "trunk" fat (TF) was derived from the sum of IAAT and ASAT: TF $=$ IAAT + ASAT

The total time for the abdominal segmentation was approximately 40-60 minutes per dataset. The SliceOmatic analysis of the $T_{1}$ weighted images was performed by an independent observer (Vardis Group, London, UK).

\section{AMRATM $^{\text {TM }}$ Profiler Image Analysis}

Image analysis was performed using $\mathrm{AMRA}^{\mathrm{TM}}$ Profiler (Advanced MR Analytics $A B$, Linköping, Sweden) as previously described $(22,23)$, with some modifications.

Water and fat images were calculated using a two-step process. First, an initial set of water and fat images was calculated using the first set of opposite phase (TE $=2.3$ $\mathrm{ms}$ ) and in-phase (TE $=4.6 \mathrm{~ms}$ ) images, using the inverse gradient method $(26,27)$. In order to correct for R2*-effects and the fat signal spectrum, a final set of water-fat images were then calculated using all echoes with an in-house implementation of IDEAL reconstruction $(28,29)$.

To obtain quantitative fat images, the water and fat image pairs were calibrated using the method described in 20,30 . In summary, a quantitative fat image is computed based on pure adipose tissue as an internal signal reference. Hence, the signal intensity level in a given fat image voxel is related to the intensity in pure adipose tissue, which is given the value 1 , corresponding to $100 \%$ adipose tissue. 
The IAAT and ASAT compartments were automatically segmented using non-rigid image registration of a library of manually segmented atlases as described in (22). A library of 10 atlases representing a range of body shapes with manually segmented labels for IAAT, arms, and internal non-visceral adipose tissue was used. A combination of atlas-based segmentation and morphological operations was used to remove the arms. In order to limit variability due to breathing, AMRA ${ }^{\mathrm{TM}}$ Profiler uses top of femoral head and top of vertebrae T9, as lower and upper limits of the abdominal region in the segmentation of ASAT.

In order to further improve the segmentation performance for a larger variation of body shapes, the result from the atlas-based registrations was interpreted as a probability map (31) for each fat compartment, where 1 means that all atlases agree on the classification of adipose tissue and 0 means that no atlas agrees. The final definition of each fat compartment was obtained by applying a threshold value to the probability map of each compartment label.

A quick visual inspection of the segmentation of each compartment was performed. In this step, the operator can observe the automated segmentation suggested by the computer and, if necessary, locally adjust the default threshold of the probability map in order to interactively change the final segmentation. To assess inter- and intraoperator variability, the manual interaction was performed three times by three different operators. All operators were employees at AMRA and trained to perform this task.

To enable a direct comparison to the conventional SliceOmatic analysis, the volumes were manually cropped at approximately the same levels as the uppermost and lowest slices used to define the abdominal region in the SliceOmatic analysis. 
Finally, the calibrated fat signal was integrated within each segmented compartment. A scaling with the voxel volume then gave the total volume of adipose tissue within each compartment.

\section{Statistical analysis}

All data are presented as means \pm SD. Statistical analysis was performed in Microsoft Excel 2011 (v. 14.2.4) and SPSS (v. 22) for the inter- and intra-observer variability ICC. The Shapiro-Wilk test was performed to test normal distribution of the difference between the two methods. Agreement between techniques was tested with Bland and Altman's method. Significance of the difference was determined by a two-tailed paired Student's t-test. The inter- and intra-observer variability were assessed using the coefficient of variation (CoV) and the intra-class correlation coefficient (ICC) using a Two-way mixed, absolute agreement model and single measures. Inter-observer CoV was computed for each observer as the quotient between the standard deviation of the three observations and the mean observation and then averaged over all 23 subjects. The intra-observer CoV was computed as the standard deviation of the three observers mean observations divided by the total mean and then averaged over all 23 subjects. The intra-observer ICC was computed for each operator separately. The inter-observer ICC was computed between the mean values of each operator. 


\section{Results}

Typical MR images and their respective image analysis results from one of the volunteers can be seen in Figure 1. Quantitative measurements of IAAT, ASAT and total trunk fat were extracted from such images, using the standard SliceOmatic and the AMRA ${ }^{\mathrm{TM}}$ Profiler (Table 1). On average it took over 40 minutes for the abdominal region from the whole body dataset to be fully analysed by SliceOmatic, necessitating continuous manual input from an expert operator. The AMRATM Profiler required less than three minutes of manual intervention. The automated intensity inhomogeneity correction and calibration of the fat image volume took approximately 10 minutes and the atlas-based segmentation took approximately 7 minutes per atlas on a standard PC.

The Shapiro-Wilk test showed no significant deviation from a normal distribution for the differences in IAAT, ASAT and trunk fat measurements $(p=0.077, p=0.147$ and $p=0.159$ respectively). There was no significant difference in the amount of IAAT measured using AMRA ${ }^{\mathrm{TM}}$ Profiler compared with the conventional SliceOmatic analysis (AMRATM Profiler $4.73 \pm 1.99$ vs SliceOmatic $4.73 \pm 1.75$ litres, $p=0.97$ ). The difference in quantification of ASAT was $10.39 \pm 5.38$ (AMRATM Profiler) vs $9.78 \pm$ 5.36 litres (SliceOmatic), $p<0.001$ and for trunk fat $15.12 \pm 5.74$ (AMRA ${ }^{\mathrm{TM}}$ Profiler ) vs $14.50 \pm 5.50$ litres (SliceOmatic), $p=0.005$.

Excellent agreement between the two methods was observed for all fat depots (Figure 2). For IAAT the 95\% limits of agreement were -1.06 - 1.07 (Figure 2a). Similar findings were observed with ASAT, where the $95 \%$ limits of agreement were 0.36 - 1.60 (Figure 2b). For trunk fat, the linear regression coefficient was 1.03 with 
an offset of 0.19 litres and the $95 \%$ limits of agreements were $-1.26-2.50$ litres (Figure 2c). However, on average the AMRA rapid semi-automated system volume estimates of ASAT and trunk fat were numerically larger, though not significantly, than the SliceOmatic analysis (6.3\% for ASAT and $4.3 \%$ for trunk fat). The measurement of IAAT was very similar between the two methods ( $0.1 \%$ for IAAT). A linear regression analysis of the Bland-Altman plots showed a significant linear regression coefficient of $0.134(p=0.029)$ for IAAT. For ASAT and trunk fat, there was no significant linear regression ( $p=0.834$ and $p=0.248$ respectively). Neither of the errors in IAAT or ASAT showed any significant correlation to the amount of trunk fat $(p=0.248$ and $p=0.335$ respectively).

The intra-observer CoV was $0.9 \%, 1.5 \%$, and $2.4 \%$ for operators $1-3$ respectively (average $1.6 \%$ ) for IAAT and $0.6 \%, 1.1 \%$ and $1.6 \%$ respectively (average $1.1 \%$ ) for ASAT. The intra-observer ICC was $1.000,0.999$, and 0.996 for operators 1-3 respectively (average 0.998 ) for IAAT and $1.000,0.999$, and 0.998 for operators $1-3$ respectively (average 0.999 ) for ASAT. The inter-observer CoV was $1.4 \%$ for IAAT and $1.2 \%$ for ASAT. The inter-observer ICC was 0.999 for both IAAT and ASAT. 


\section{Discussion}

Rapid scanning protocols as well as automated image analysis are essential in large population studies where in vivo imaging modalities are becoming the norm. This study shows that quantification of central obesity, including IAAT ("visceral fat") and ASAT can be done using a rapid semi-automated quantification method of MR images acquired with a very rapid multi-point Dixon protocol. Furthermore, the correlation to the current gold-standard semi-automated segmentation program (SliceOmatic) was extremely high for all fat depots. Also the agreement between the two methods of quantification was extremely high.

The design of the study was such that not only differences in analysis method were a factor, but also the MRI acquisition protocol. Whilst it might seem counter-intuitive to both acquire the data using different MRI sequences (T1 vs 3D-Dixon) with the patient in a different position (Prone vs supine); it was decided that a true test of the standard vs the AMRA ${ }^{\mathrm{TM}}$ Profiler, must ensure that the optimal and validated protocol should be used in each instance, so as to minimise potential bias for any given method. Given the variation in acquisition and analysis, it is perhaps more impressive that the agreement in measurement particular of IAAT is so high. Indeed, the strong linear correlation and excellent agreement between the SliceOmatic and AMRA ${ }^{T M}$ Profiler results indicates that the latter can be reliably used for quantification of IAAT, ASAT and total trunk fat. Moreover, given that the standard segmentation technique takes $>40$ minutes per subject for a trained operator, making it unfeasible for large population studies, the short analysis time of the AMRA ${ }^{\text {TM }}$ Profiler, less than three minutes per subject, opens a realistic possibility for the analysis of MRI data sets 
from large cohort studies. The computation time for the automated processing, of course, depends on implementation and hardware. Parallel computing e.g. using a GPU implementation or multi-core CPU could of course reduce the current computation time.

The linear regression coefficient was close to one for both compartments, though the AMRA $^{\mathrm{TM}}$ Profiler volume estimates were numerically larger for ASAT, though not significantly, than the SliceOmatic analysis. The differences between techniques were independent of the $\mathrm{BMI}$ and/or the total body fat content of the volunteers. Therefore it is possible that the methods of defining which slices from the whole body dataset to equate to the abdominal compartment generally used (the top of the liver to top of femoral head) could account for this difference rather than a difference between the analysis methods per se. There was, however, a positive linear correlation between the differences in IAAT measures and the IAAT volume, indicating that for subjects with more IAAT, the AMRATM Profiler tends to give smaller IAAT estimates than the reference method. The different acquisition approaches necessitated identifying the selecting matching top and bottom slices from an axial acquisition with relatively thick slices and inter-slice gaps and from a 3D dataset. A small mismatch particularly in the lower slices containing the femoral heads and could have a significant impact of the amount of subcutaneous adipose tissue included (but not internal adipose tissue), since this covers the area where anatomically a small change in position can result in a substantial difference in subcutaneous fat content. 
A limitation with the AMRA $^{T M}$ Profiler is that it requires fat-water separated or complex-valued Dixon images, and can therefore not be used for analysing already existing data acquired with, for example, a more traditional T1-weighted protocol. However, the proposed rapid Dixon protocol has several advantages compared to the more traditional T1-weigthed protocol used as reference in this study. First of all, the close to isotropic image resolution, in combination with the breath-hold technique, gives a complete three-dimensional data volume rather than a stack of more or less independent two-dimensional image slices. This significantly simplifies the use of tree-dimensional image processing, which facilitates volumetric measurements of also other anatomical structures and organs. Secondly, the calibrated fat image is specifically sensitive to fat, which is not the case for T1-weighted images. A calibrated fat image enables quantification also of diffuse fat infiltration e.g. in muscles and internal organs.

It should also be stressed that the AMRA'M Profiler gives an objective, userindependent quantification of the fat signal. Only the anatomical definition of the compartment of interest is subject to segmentation as well as inspection and manual interaction. This is important e.g. in the visceral compartment where intestinal content easily can be mistaken for adipose tissue. The excellent inter- and intra-observer ICC and the very low inter- and intra-observer variability shown in this study confirm this. The CoV for the intra-observer comparison of $1.6 \%$ for IAAT and $1.1 \%$ for ASAT can be compared to values reported in an earlier study (32) where the investigated method (Hippo Fat) had a CoV of $7.25 \%$ for IAAT and $1.77 \%$ for ASAT and the SliceOmatic analysis had a CoV of $4.53 \%$ for IAAT and $1.85 \%$ for ASAT. 
The use of a quantitative fat image also means that, as opposed to methods based on classification of individual voxels into adipose or non-adipose tissue such as (1416), the method used here is much less affected by partial volume effects (21) since also the fat in voxels containing a mix of adipose and non-adipose tissue will be included. Estimation errors caused by partial volume effects increase with lower resolution, which is a consequence of rapid whole body acquisition. Furthermore, the AMRA $^{T M}$ Profiler used in this study has also been used for compartmental muscle volume measurements (33) which also is a relevant factor in metabolic studies. 


\section{Acknowledgements}

ELT and JDB gratefully acknowledge funding from the UK Medical Research Council. We would like to thank Dr AP Goldstone and Giuliana Durighel for their contribution in the recruitment and scanning of volunteers.

\section{Conflict of Interest Statement}

ELT, JF and JDB have no conflict of interest to declare. MB, TR, JR and ODL are stock holders in Advanced MR Analytics (AMRA) AB. 


\section{References}

1. Neeland IJ, Ayers CR, Rohatgi AK, Turer AT, Berry JD, Das SR, Vega GL, Khera A, McGuire DK, Grundy SM, de Lemos JA. Associations of visceral and abdominal subcutaneous adipose tissue with markers of cardiac and metabolic risk in obese adults. Obesity. 2013; 21: E439-E47.

2. Cerhan JR, Moore SC, Jacobs EJ, Kitahara CM, Rosenberg PS, Adami H-O, Ebbert JO, English DR, Gapstur SM, Giles GG, Horn-Ross PL, Park Y, Patel AV, Robien K, Weiderpass E, Willett WC, Wolk A, Zeleniuch-Jacquotte A, Hartge P, Bernstein L, Berrington de Gonzalez A. A pooled analysis of waist circumference and mortality in 650,000 adults. Mayo Clinic Proc. 2014; 89: 335-45.

3. Prentice AM, Jebb SA. Beyond body mass index. Obesity Reviews. 2001; 2: 1417.

4. Thomas EL, Frost G, Taylor-Robinson SD, Bell JD. Excess body fat in obese and normal-weight subjects. Nutr Res Rev. 2012; 25: 150-61.

5. Thomas EL, Parkinson JR, Goldstone AP, McCarthy JP, Fitzpatrick JA, Durighel G, Taylor-Robinson SD, Bell JD. The missing risk: MRI and MRS phenotyping of abdominal adiposity and ectopic fat. Obesity. 2012; 20: 76-87.

6. Thomas EL, Saeed N, Hajnal JV, Brynes A, Goldstone AP, Frost G, Bell JD. Magnetic resonance imaging of total body fat. J App Physiol. 1998; 85: 1778-85.

7. Machann J, Schnoedt B, Schick F, Thamer C, Haap M, Haring HU, Claussen CD, Stumvoll M, Fritsche A, Schick F. Standardized assessment of whole body adipose tissue topography by MRI. J Magn Reson Imaging 2005; 21: 455-62. 
8. Chandra A, Neeland IJ, Berry JD, Ayers CR, Rohatgi A, Das SR, Khera A, McGuire DK, de Lemos JA, Turer AT. The relationship of body mass and fat distribution with incident hypertension: observations from the Dallas Heart Study. J Am Coll Cardiol. 2014; 64:997-1002.

9. Fox CS, Massaro JM, Hoffmann U, Pou KM, Maurovich-Horvat P, Liu CY, Vasan RS, Murabito JM, Meigs JB, Cupples LA, D'Agostino RB Sr, O'Donnell CJ. Abdominal visceral and subcutaneous adipose tissue compartments: association with metabolic risk factors in the Framingham Heart Study. Circulation. 2007; 116:39-48.

10. Shah RV, Murthy VL, Abbasi SA, Blankstein R, Kwong RY, Goldfine AB, JeroschHerold M, Lima JA, Ding J, Allison MA. Visceral Adiposity and the Risk of Metabolic Syndrome Across Body Mass Index: The MESA Study. JACC Cardiovasc Imaging. 2014;7(12):1221-1235.

11. Thomas EL, Bell JD. Influence of undersampling on magnetic resonance imaging measurements of intra-abdominal adipose tissue. Int J Obes Relat Metab Disord. $2003 ; 27: 211-8$.

12. Bonekamp S, Ghosh P, Crawford S, Solga SF, Horska A, Brancati FL, Diehl AM, Smith S, Clark JM.. Quantitative comparison and evaluation of software packages for assessment of abdominal adipose tissue distribution by magnetic resonance imaging. Int J Obes 2008; 32: 100-11.

13. Fall T, Ingelsson E. Genome-wide association studies of obesity and metabolic syndrome. Mol Cell Endocrinol. 2014; 382: 740-57. 
14. Kullberg J, Karlsson AK, Dahlgren J, Stokland E, Svensson PA. Adipose tissue distribution in children: Automated quantification using water and fat MRI. J Magn Reson Imaging. 2010; 32: 204-10.

15. Poonawalla AH, Sjoberg BP, Rehm JL, Hernando D, Hines CD, Irarrazaval P, Reeder SB. Adipose tissue MRI for quantitative measurement of central obesity. J Magn Reson Imaging. 2013; 37: 707-16.

16. Thörmer G, Bertram HH, Garnov N, Peter V, Schütz T, Shang E, Blüher M, Kahn T, Busse H. Software for automated MRI-based quantification of abdominal fat and preliminary evaluation in morbidly obese patients. J Magn Reson Imaging. 2013; 37: 1144-50.

17. Wald D, Teucher B, Dinkel J, Kaaks R, Delorme S, Boeing H, Seidensaal K, Meinzer HP, Heimann T. Automatic quantification of subcutaneous and visceral adipose tissue from whole-body magnetic resonance images suitable for large cohort studies. J Magn Reson Imaging. 2012; 36:1421-34

18. Müller HP, Raudies F, Unrath A, Neumann H, Ludolph AC, Kassubek J. Quantification of human body fat tissue percentage by MRI. NMR Biomed. 2011; 24:17-24

19. Addeman BT, Kutty S, Perkins TG, Soliman AS, Wiens CN, McCurdy CM, Beaton MD, Hegele RA, McKenzie CA. Validation of volumetric and single-slice MRI adipose analysis using a novel fully automated segmentation method. J Magn Reson Imaging. 2015; 41: 233-41 
20. Würslin C, Machann J, Rempp H, Claussen C, Yang B, Schick F. Topography mapping of whole body adipose tissue using A fully automated and standardized procedure. J Magn Reson Imaging. 2010; 31: 430-9

21. Ludwig UA, Klausmann F, Baumann S, Honal M, Hövener JB, König D, Deibert $P$, Büchert M. Whole-body MRI-based fat quantification: A comparison to air displacement plethysmography. J Magn Reson Imaging. 2014; 40: 1437-44

22. Leinhard OD, Johansson A, Rydell J, Smedby O, Nystrom F, Lundberg P. Quantitative Abdominal Fat Estimation Using MRI. Int Conf Pattern Recognition. 2008; $19: 2137-40$.

23. Erlingsson S, Herard S, Dahlqvist $O$, Lindström T, Länne T, Borga M, Nystrom FH; Fast Food Study Group. Men develop more intraabdominal obesity and signs of the metabolic syndrome after hyperalimentation than women. Metabolism 2009; 58: 995-1001.

24. Dixon W. Simple proton spectroscopic imaging. Radiology 1984; 153: 189-94.

25. Ostberg JE, Thomas EL, Hamilton G, Attar MJH, Bell JD, Conway GS. Excess visceral and hepatic adipose tissue in Turner syndrome determined by magnetic resonance imaging: estrogen deficiency associated with hepatic adipose content. J Clin Endocrinol Metab. 2005; 90: 2631-5.

26. Rydell J, Knutsson H, Pettersson J, Johansson A, Farnebäck G, Dahlqvist O, Lundberg P, Nyström F, Borga M. Phase Sensitive Reconstruction for Water/Fat Separation in MR Imaging Using Inverse Gradient. Med Image Comput Comput Assist Interv 2007; 10: 210-8. 
27. Rydell J, Johansson A, Leinard OD, Knutsson H, Farnebäck G, Lundberg P. Three Dimensional Phase Sensitive Reconstruction for Water/Fat Separation in MR Imaging using Inverse Gradient. Proc. Int. Soc. Magn. Reson. Med. 2008; 1519.

28. Reeder SB, Pineda AR, Wen Z, Shimakawa A, Yu H, Brittain JH, Gold GE, Beaulieu $\mathrm{CH}$, Pelc NJ. Iterative decomposition of water and fat with echo asymmetry and least-squares estimation (IDEAL): application with fast spin-echo imaging. Magn Reson Med. 2005; 54: 636-44.

29. Hernando D, Hines CDG, Yu H, Reeder SB. Addressing phase errors in fat-water imaging using a mixed magnitude/complex fitting method. Magn Reson Med. 2012; 67: 638-44.

30. Romu T, Borga M, Dahlqvist Leinhard O. MANA - Multi scale adaptive normalized averaging. 2011 IEEE Int Symp Biomed Imaging: From Nano to Macro. 2011; 3614.25 .

31. Svarer C, Madsen K, Hasselbalch SG, Pinborg LH, Haugbøl S, Frøkjær VG, Holm S, Paulson OB, Knudsen GM. MR-based automatic delineation of volumes of interest in human brain PET images using probability maps. Neuro Image. 2005; 24: 969-79.

32. Demerath EW, Ritter KJ, Couch WA, et al. Validity of a new automated software program for visceral adipose tissue estimation. International journal of obesity. 2007; 31:285-291.

33. Thomas MS, Newman D, Leinhard OD, Kasmai B, Greenwood R, Malcolm PN, Karlsson A, Rosander J, Borga M, Toms AP. Test-retest reliability of automated 
whole body and compartmental muscle volume measurements on a wide bore 3T MR system. Eur Radiol. 2014; 24: 2279-91. 


\section{Figure Captions}

Figure 1. MR images from typical volunteer. (a) shows the calibrated fat image with intra-abdominal and subcutaneous segmentations made by the AMRATM Profiler overlaid in red and green respectively. The right panel show the approximately corresponding transverse slice (b) from the same subject analysed by SliceOmatic. In this example both images were acquired with the volunteer positioned in a supine position to make the images more readily comparable.

Figure 2. Bland and Altman plots describing agreement between fat volumes measured using SliceOmatic and the AMRA ${ }^{\mathrm{TM}}$ Profiler method for (a) intra-abdominal adipose tissue (IAAT), (b) abdominal subcutaneous adipose tissue (ASAT) and (c) Total trunk adipose tissue 
Table 1. Measurements of IAAT, ASAT and Trunk AT with the two analysis methods.

\begin{tabular}{|c|c|c|c|c|c|c|}
\hline & \multicolumn{2}{|c|}{ IAAT (I) } & \multicolumn{2}{|c|}{ ASAT (I) } & \multicolumn{2}{|c|}{ Trunk AT (I) } \\
\hline Subject \# & SliceOmatic & AMRA & SliceOmatic & AMRA & SliceOmatic & AMRA \\
\hline 1 & 1.30 & 1.16 & 11.73 & 11.48 & 13.03 & 12.63 \\
\hline 2 & 5.85 & 6.70 & 4.44 & 5.38 & 10.29 & 12.08 \\
\hline 3 & 6.24 & 6.28 & 11.71 & 12.52 & 17.94 & 18.80 \\
\hline 4 & 4.12 & 4.03 & 20.67 & 21.63 & 24.79 & 25.66 \\
\hline 5 & 5.50 & 5.20 & 5.29 & 5.72 & 10.79 & 10.92 \\
\hline 6 & 3.36 & 3.33 & 2.94 & 3.46 & 6.30 & 6.79 \\
\hline 7 & 5.14 & 4.74 & 5.82 & 6.56 & 10.96 & 11.29 \\
\hline 8 & 4.00 & 3.48 & 7.79 & 7.90 & 11.80 & 11.38 \\
\hline 9 & 3.74 & 3.13 & 15.39 & 15.42 & 19.13 & 18.56 \\
\hline 10 & 7.23 & 8.69 & 7.73 & 9.76 & 14.96 & 18.45 \\
\hline 11 & 2.51 & 2.16 & 7.84 & 8.52 & 10.36 & 10.68 \\
\hline 12 & 4.18 & 3.40 & 10.16 & 9.74 & 14.34 & 13.14 \\
\hline 13 & 7.38 & 7.14 & 9.54 & 10.75 & 16.92 & 17.89 \\
\hline 14 & 2.09 & 2.36 & 7.21 & 7.93 & 9.30 & 10.29 \\
\hline 15 & 6.66 & 6.52 & 9.92 & 10.22 & 16.58 & 16.74 \\
\hline 16 & 5.72 & 5.00 & 7.35 & 7.75 & 13.06 & 12.75 \\
\hline 17 & 4.09 & 4.10 & 10.86 & 11.75 & 14.95 & 15.84 \\
\hline 18 & 4.31 & 4.50 & 4.30 & 4.92 & 8.62 & 9.42 \\
\hline 19 & 6.06 & 6.03 & 11.27 & 11.67 & 17.32 & 17.70 \\
\hline 20 & 7.75 & 8.37 & 6.05 & 6.76 & 13.81 & 15.12 \\
\hline 21 & 3.39 & 3.53 & 18.71 & 19.64 & 22.09 & 23.17 \\
\hline 22 & 2.87 & 2.81 & 4.71 & 5.40 & 7.58 & 8.21 \\
\hline
\end{tabular}


Volumes of intra-abdominal (IAAT), abdominal subcutaneous (ASAT) and total trunk adipose tissue measured in litres for each volunteer measured using SliceOmatic and AMRA ${ }^{\mathrm{TM}}$ Profiler. 
Table 2. Measurements of IAAT and ASAT using AMRA ${ }^{\text {TM }}$ Profiler by three different operators

\begin{tabular}{|c|c|c|c|c|c|c|}
\hline & \multicolumn{3}{|c|}{ IAAT (I) } & \multicolumn{3}{|c|}{ ASAT (I) } \\
\hline Subject \# & Op. 1 & Op. 2 & Op. 3 & Op. 1 & Op. 2 & Op. 3 \\
\hline 1 & 7.58 & 7.67 & 7.66 & 12.59 & 12.67 & 12.72 \\
\hline 2 & 7.31 & 7.36 & 7.32 & 12.15 & 12.22 & 12.26 \\
\hline 3 & 3.68 & 3.78 & 3.88 & 9.43 & 9.39 & 9.54 \\
\hline 4 & 2.53 & 2.60 & 2.56 & 8.40 & 8.38 & 8.60 \\
\hline 5 & 1.32 & 1.29 & 1.29 & 11.08 & 10.98 & 11.22 \\
\hline 6 & 5.73 & 5.77 & 5.73 & 5.95 & 5.83 & 6.05 \\
\hline 7 & 9.28 & 9.41 & 9.23 & 7.72 & 7.75 & 7.79 \\
\hline 8 & 3.84 & 3.94 & 3.91 & 3.63 & 3.57 & 3.56 \\
\hline 9 & 6.73 & 6.86 & 6.73 & 5.39 & 5.26 & 5.36 \\
\hline 10 & 7.05 & 7.18 & 7.04 & 11.51 & 11.44 & 11.59 \\
\hline 11 & 6.78 & 6.95 & 6.79 & 13.25 & 13.24 & 13.23 \\
\hline 12 & 4.45 & 4.45 & 4.39 & 21.63 & 21.67 & 22.10 \\
\hline 13 & 4.35 & 4.53 & 4.49 & 19.39 & 18.86 & 19.03 \\
\hline 14 & 3.15 & 3.18 & 3.11 & 15.80 & 15.74 & 15.70 \\
\hline 15 & 9.61 & 9.76 & 9.50 & 10.61 & 10.51 & 10.52 \\
\hline 16 & 4.36 & 4.39 & 4.47 & 13.66 & 13.65 & 13.87 \\
\hline 17 & 3.34 & 3.40 & 3.37 & 5.56 & 5.57 & 5.61 \\
\hline 18 & 2.35 & 2.43 & 2.54 & 9.35 & 9.35 & 9.58 \\
\hline 19 & 4.98 & 5.07 & 5.02 & 4.88 & 4.84 & 4.94 \\
\hline 20 & 5.38 & 5.45 & 5.28 & 7.44 & 7.34 & 7.38 \\
\hline 21 & 4.43 & 4.52 & 4.56 & 12.93 & 12.91 & 13.12 \\
\hline 22 & 6.96 & 7.38 & 7.14 & 24.23 & 23.38 & 22.92 \\
\hline 23 & 5.11 & 5.18 & 5.30 & 6.50 & 6.44 & 6.42 \\
\hline
\end{tabular}

Volumes (means \pm SD) of intra-abdominal (IAAT), abdominal subcutaneous (ASAT) measured three times by three different operators. The coefficient of variation (CoV) for the intra-observer comparison was $1.6 \%$ for IAAT and $0.51 .1 \%$ for ASAT. The inter-subject CoV for IAAT and ASAT was $1.6 \%$ and $1.1 \%$ respectively. 

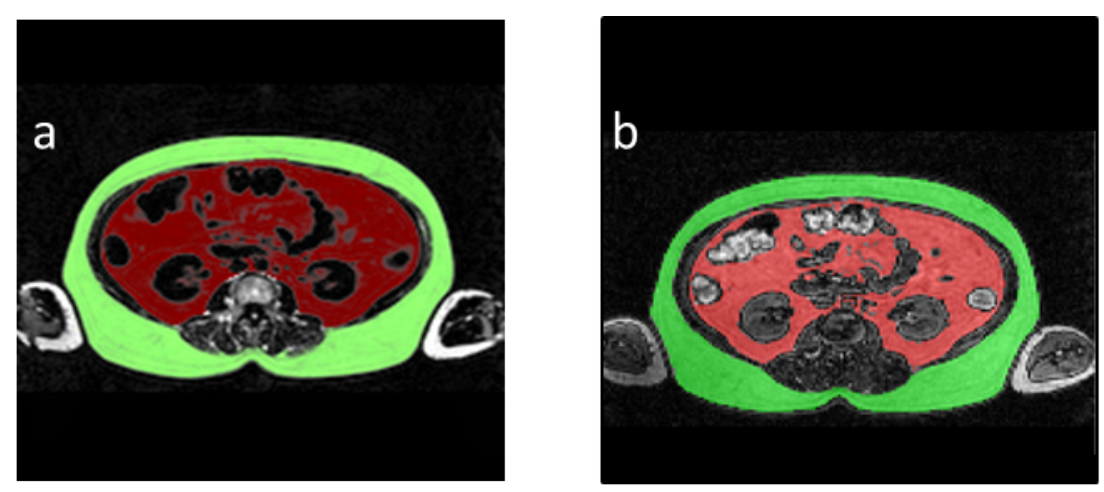
Figure 2

a

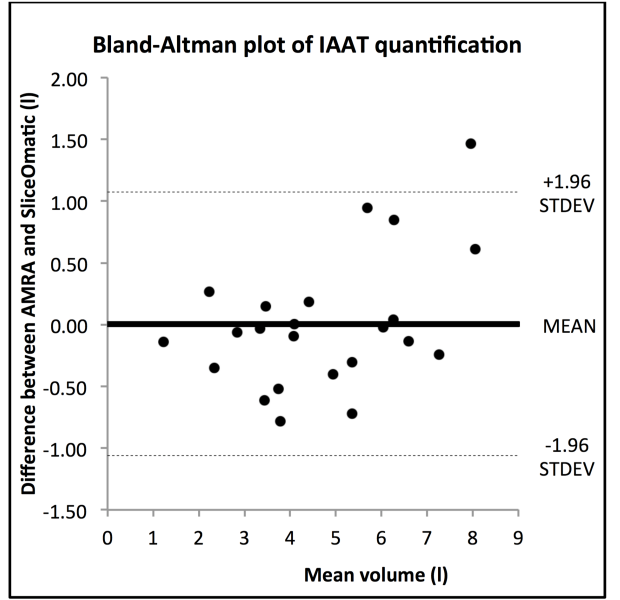

b

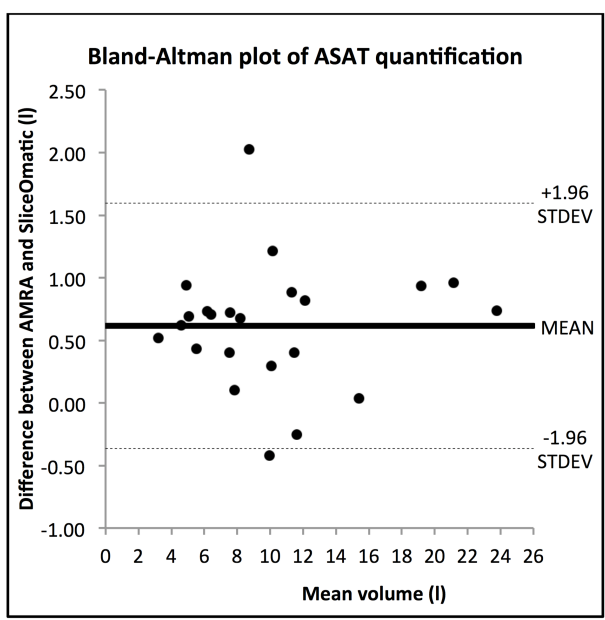

c

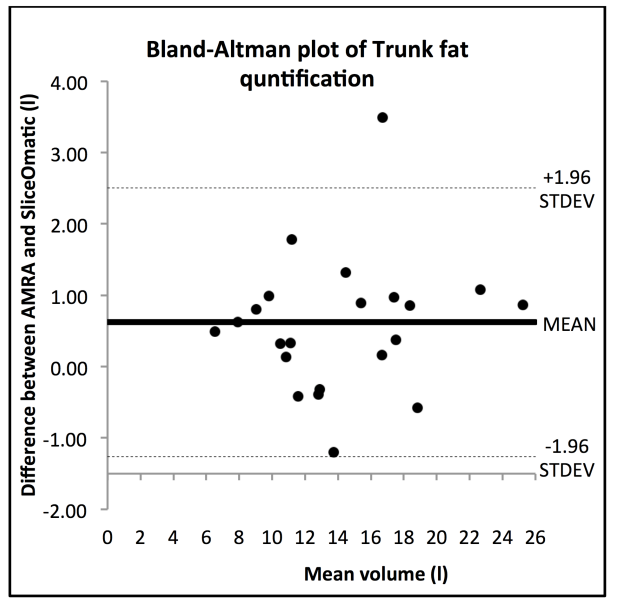

